\title{
A Possible Antioxidant Role for Vitamin D in Soccer Players: A Retrospective Analysis of Psychophysical Stress Markers in a Professional Team
}

\author{
Davide Ferrari ${ }^{1,2, *} \mathbb{0}$, Giovanni Lombardi ${ }^{3,4}{ }^{\mathbb{C}}$, Marta Strollo ${ }^{2}$, Marina Pontillo ${ }^{2}$, \\ Andrea Motta ${ }^{2}$ and Massimo Locatelli ${ }^{2}$ \\ 1 SCVSA Department, University of Parma, 43125 Parma, Italy \\ 2 Laboratory Medicine Service, San Raffaele Hospital, 20132 Milano, Italy; strollo.marta@hsr.it (M.S.); \\ pontillo.marina@hsr.it (M.P.); motta.andrea@hsr.it (A.M.); locatelli.massimo@hsr.it (M.L.) \\ 3 Laboratory of Experimental Biochemistry and Molecular Biology, IRCCS Istituto Ortopedico Galeazzi, \\ 20161 Milano, Italy; giovanni.lombardi@grupposandonato.it \\ 4 Department of Athletics, Strength and Conditioning, Poznań University of Physical Education, 61-871 \\ Poznań, Poland \\ * Correspondence: davide.ferrari@unipr.it; Tel.: +39-0521-906633; Fax: +39-0521-905151
}

Received: 8 April 2020; Accepted: 14 May 2020; Published: 16 May 2020

\begin{abstract}
The health benefits of physical activity are recognized, however, high levels of exercise may lead to metabolic pathway imbalances that could evolve into pathological conditions like the increased risk of neurological disease observed in professional athletes. We analyzed the plasma/serum levels of 29 athletes from a professional soccer team playing in the Italian first league and tested the levels of psychophysical stress markers (vitamin D, creatine kinase, reactive oxygen species (ROS) and testosterone/cortisol ratio) during a period of 13 months. The testosterone/cortisol ratio was consistent with an appropriate training program. However, most of the athletes showed high levels of creatine kinase and ROS. Despite the large outdoor activity, vitamin D values were often below the sufficiency level and, during the "vitamin D winter", comparable with those of the general population. Interestingly, high vitamin D values seemed to be associated to low levels of ROS. Based on the results of our study we proposed a vitamin D supplementation as a general practice for people who perform high levels of physical exercise. Beside the known effect on calcium and phosphate homeostasis, vitamin D supplementation should mitigate the high reactivity of ROS which might be correlated to higher risk of neurodegenerative diseases observed in professional athletes.
\end{abstract}

Keywords: overtraining; creatine kinase; vitamin D; ROS; testosterone; cortisol; SOD1

\section{Introduction}

Physical activity and exercise training are recognized to provide a range of significant benefits associated to both physical and mental health [1,2]. Nevertheless, excessive exercise (EE) may lead to an increased risk of heart dysfunctions as well as altered biological, neurochemical, and hormonal regulation mechanisms [3]. This is common in professional athletes whose training involves an overload period which is often not complemented by an adequate recovery. As a consequence, depending on the time needed to restore performance capacity, athletes may experience overtraining/overreaching syndrome [4] which is frequently associated to catabolic and anabolic imbalance involving skeletal muscle proteins, the neuroendocrine system, and the autonomic nervous system $[5,6]$. Furthermore, EE has been associated to sport-related skeletal muscle injuries due to repetitive sarcolemma micro-damages and altered calcium homeostasis, bony stress fractures due aberrant loads and accelerated bone turnover, and acute macro-trauma especially in contact sports [7]. If contact sports and musculoskeletal injuries 
might be seen as an obvious connection of events, a less evident correlation was observed lately between sports and neurodegenerative diseases [8]. Recent studies showed that former professional athletes, who participated in contact sports, had an increased risks of impaired cognitive function and dementia, Parkinson's disease, Alzheimer disease and amyotrophic lateral sclerosis (ALS) [8-10]. However, besides the statistical significance, no clear evidence explaining the association between head trauma and neurodegenerative diseases has been proposed. Furthermore, conflicting results have been published showing an association between high-level chronic physical activity and ALS regardless of any traumatic events [11]. ALS, a fatal adult-onset neurodegenerative disorder often associated with professional sports and soccer in particular [12,13], is characterized by the progressive loss of motor neurons in the brain, brainstem, and spinal cord, which brings paralysis and death within a few years from diagnosis $[11,14]$. Recent studies showed that the motor neurons impairment in ALS is often associated with the protein misfolding and deposition of superoxide dismutase 1 (SOD1), into insoluble aggregates, likely caused by a structural destabilization induced by gene mutations and/or oxidative damage [15].

Thus, although many studies have shown that "a certain level of exercise is good", more seems not to be necessarily better and too much might become deleterious [2].

During a season, soccer players face up to several matches in a congested schedule, with as little as 3 days of recovery in between. In this situation a complete recovery is not reached due to the added biochemical stress. Fatigue, indeed, may persist for days after a single match, impairing physical performance and neuromuscular functions, increasing perceptual discomfort (e.g., muscle soreness) and inducing biochemical perturbations (e.g., muscle damage, inflammatory and immunological markers) [16]. For instance, blood creatine kinase (CK) activity remained significantly higher during the 72 h-recovery period [17,18] and the immune function remained altered in 48 h-post match [19]. Accordingly, the accumulation of muscle damage, inflammatory and immune perturbations triggered by consecutive matches and the daily training sessions may hinder the recovery and, consequently, limit the athletes' readiness and increase the risk of injury [20-22].

We retrospectively analyzed the plasma/serum levels of different psychophysical stress markers such as the testosterone (T) to cortisol (C) ratio [23], vitamin D (vitD) [24,25], creatine kinase (CK) [24] and reactive oxygen species (ROS) [26], in an elite soccer team of the Italian first league during an entire season with the aim of determining the seasonal changes and the eventual association with pathological conditions or injuries that emerged during the observation period.

\section{Materials and Methods}

\subsection{Study Cohort}

The entire squad of soccer players, made up by 29 male athletes, aged $18-40$ years (25.9 \pm 5.0 years), belonging to the A.C. Milan football team of the Italian "Serie A" were included in this retrospective observational study. The averaged heights and weights were, respectively, $183.7 \pm 5.9 \mathrm{~cm}$ and $77.5 \pm$ $7.7 \mathrm{Kg}$. The team regularly trained and competed at latitudes with middle/high sun exposure (between $45^{\circ}$ and $46^{\circ} \mathrm{N}$ of latitude), even during autumn and winter. The competitive season started on 20th of August 2017 and ended on 20th of May 2018. Thus, the off-season typically takes place in June and the pre-season starts in July. All individuals involved in the study gave an informed consent to the use of their anonymously collected data for retrospective observational studies (with reference to article 9.2.j of the EU general data protection regulation 2016/679 (GDPR)), according to the San Raffaele Hospital internal policy (IOG075/2016).

No regular supplementation of vitD was followed by the team, however, a sporadic intake of vitamin $\mathrm{D}$ by single athletes cannot be excluded. 


\subsection{Samples Collection}

Blood drawings were performed on July 5th, 2017; September 13th, 2017; November 29th, 2017; January 15th, 2018; March 6th, 2018; April 24th, 2018; July 10th, 2018 and August 15th, 2018. Blood, obtained by standard venipuncture into BD-SST II Advance tube, $3.5 \mathrm{~mL}, 13 \times 75 \mathrm{~mm}$ from BD (Becton, Dickinsonand Company, NJ, USA) [27], according to the most up-to-date pre-analytical warnings, was immediately refrigerated and brought to the laboratory of the San Raffaele Hospital in Milan.

\subsection{Clinical Data}

VitD, CK activity, ROS, and T/C were evaluated in all samples. Measurements were performed, within $4 \mathrm{~h}$ from withdrawal, on a Roche COBAS 8000 [28] (Roche, Basel, Switzerland) using electrochemiluminescence immunoassays $(25(\mathrm{OH}) \mathrm{D}, \mathrm{T}$ and $\mathrm{C})$, and spectrophotometric assays (CK activity and ROS). VitD was measured as serum total 25-hydroxyvitamin D (25(OH)D) which is considered the best indicator of vitD status [29]. ROS concentrations were expressed in Carratteli units ( $\mathrm{Car} / \mathrm{U}$ ) where one Car/U corresponds to a $\mathrm{H}_{2} \mathrm{O}_{2}$ concentration of $0.08 \mathrm{mg} / 100 \mathrm{~mL}$. The $\mathrm{T} / \mathrm{C}$ ratio was calculated by dividing the two hormone levels both expressed in $\mathrm{nm} / \mathrm{L}$. All of the instrumentation was routinely checked, each month, by averaging approximately 25-28 measurements (one each working day) of standard solution at low and high concentrations.

\subsection{Statistical Analysis}

Statistical analyses and graphs were performed with the software Sigmaplot (Systat-Software, Inc. San Jose, CA, USA) and GraphPad Prism v6.01 (GraphPad Software Inc., La Jolla, CA, USA). A linear regression analysis was performed for the whole dataset and for the dataset without the outliers (values which were at least 3 standard deviations away from the mean) to investigate the relationship between each pair of psychophysical markers. Averaged values and their corresponding standard deviation intervals (STD) were also calculated at each withdrawal date.

\section{Results}

\subsection{Vitamin D}

The averaged circannual 25(OH)D variations for the 29 players were compared with that of the general population living at the same latitude [30] Figure 1, panel (A). During the "vitD winter" (November to March) the athlete group showed average 25(OH)D levels similar to those of the general population Figure 1, panel (A) whereas in the warmer season their averaged 25(OH)D levels were higher and not included in the STD intervals of the general population. Four players (players 15, 16, 20 and 24) were of African origins and showed very low averaged 25(OH)D levels Figure 1, panel (A). The 25(OH)D levels from each player, as well as their averaged values, are shown in the supplementary materials (Table S1). Of the 195 data collected only $41 \%$ were above the $30 \mathrm{ng} / \mathrm{mL}$ sufficiency threshold [30] whereas $44.6 \%$ had insufficient levels of $25(\mathrm{OH}) \mathrm{D}(20-30 \mathrm{ng} / \mathrm{mL})$ and $14.4 \%$ were 25(OH)D deficient $(<20 \mathrm{ng} / \mathrm{mL}$ ) [27]. Most of the 25(OH)D deficient measurements (18 out of 28; $64.3 \%$ ) were from the four players of African origins which showed levels between 5.7 and $20.4 \mathrm{ng} / \mathrm{mL}$ (Table S1).

As expected, the majority of the 25(OH)D insufficient/deficient levels $(<30 \mathrm{ng} / \mathrm{mL})$ were in the "vitamin D winter" (November to March) [30]. During this 5-month interval, 74.6\% of the collected blood samples were below the suggested $30 \mathrm{ng} / \mathrm{mL}$ limit. In contrast, during the warm season only $41.1 \%$ of the samples were $25(\mathrm{OH}) \mathrm{D}$ insufficient/deficient. 


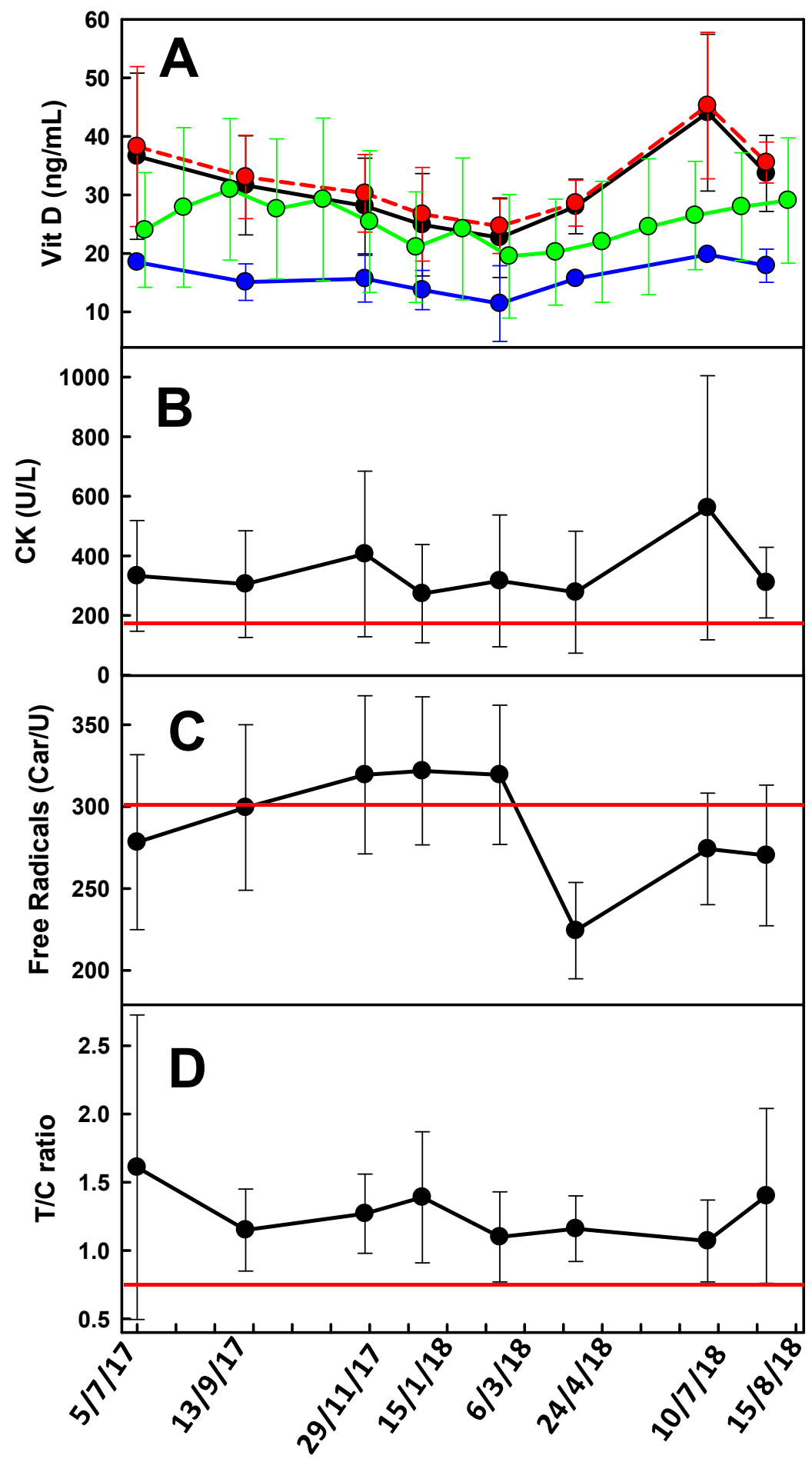

Figure 1. Seasonal variability of the averaged recorded psychophysical stress markers for the 29 soccer players involved in this study. The exact withdrawal date is shown on the $x$ axis. Panel A: Vitamin D (vitD) values for the 29 soccer players (black line), the 4 soccer players of African origins (blue line), and the 25 soccer players of non-African origins (red line). The green line represents the vitD levels of the general population living at the same latitude [30]. Panel B: creatine kinase (CK) values; the red line represents the normal clinical upper limit of $195 \mathrm{U} / \mathrm{L}$. Panel C: free radicals; the red line represents the normal clinical upper limit of $300 \mathrm{Car} / \mathrm{U}$ Panel D: testosterone (T)/cortisol (C) ratio; the red line represents the lower limit consistent with an overtraining risk $(<0.76)$. Error bars represent the standard deviation (STD) interval. 


\section{2. $C K$}

The averaged CK activities were above the normal clinical range (20-195 U/L) during the entire period of observation Figure 1, panel (B). Table S2 shows that 74.9\% of the samples analyzed exceeded the upper limit. The highest percentage of samples above the $195 \mathrm{U} / \mathrm{L}$ limit were observed both in the pre-seasons (between July and August) and at the beginning of the season (September to November), whereas in the central and final parts of the season the percentage of samples above the $195 \mathrm{U} / \mathrm{L}$ limit was between 58\% and 67\% Figure 1, panel (B). Of the 195 samples, 22 were collected when the corresponding athletes were on an injured period (Table S2) yet, 14 of them (63.6\%) had CK activity levels still above the limit (Table S2).

\subsection{Reactive Oxygen Species}

ROS exceeded the suggested upper limit of $300 \mathrm{Car} / \mathrm{U}$ in $42.6 \%$ of the measurements (Table S3). The highest percentages of athletes above the limits were recorded in the central part of the season: November 29th, 2017 (59.2\%), January 15th, 2018 (72.4\%) and March 6th, 2018 (63.0\%) Figure 1, panel (C). Of the 22 blood samples taken from soccer players on an injury period, only 3 (13.6\%) exceeded the normal range.

\subsection{T/C Ratio}

The averaged $\mathrm{T} / \mathrm{C}$ ratios were above the 0.76 level, considered as the threshold below which there is a risk of overtraining [31], for the whole season Figure 1, panel (D). Of the 195 collected samples, 183 $(93.8 \%)$ were in the normal range whereas only one athlete, at the beginning of the season (July, 5th, 2017), showed a T/C ratio $<0.50$ consistent with a high risk of overtraining (Table S4). Ten samples showed T/C levels between 0.58 and 0.75 consistent with a moderate risk of overtraining and only one sample was between 0.50 and 0.57 , thus consistent with a substantial overtraining risk. Of the 22 blood samples taken from soccer players on an injury period, only one (4.5\%) was below the 0.76 threshold.

\subsection{Correlation between Psychophysical Markers}

Table 1 shows the parameters obtained from the analysis of the linear correlations between each pair of psychophysical markers Figure 2. Among the six couples analyzed $(25(\mathrm{OH}) \mathrm{D}$ vs. ROS; $25(\mathrm{OH}) \mathrm{D}$ vs. T/C; $25(\mathrm{OH}) \mathrm{D}$ vs. CK; ROS vs. T/C; ROS vs. CK; T/C vs. CK) we observed a strong correlation between $25(\mathrm{OH}) \mathrm{D}$ and ROS (slope: -1.03 and $P=0.003$ ) and a weaker, yet significant, correlation between $25(\mathrm{OH}) \mathrm{D}$ and T/C (slope: -0.01 and $P=0.009)$. All of the other combinations showed no significant deviation from horizontal (Table 1). The same analysis was performed without outliers: the results confirmed the previous observation with the exception of the correlation between $25(\mathrm{OH}) \mathrm{D}$ and $\mathrm{T} / \mathrm{C}$ which showed no significant deviation from horizontal $(P=0.105$, data not shown).

Table 1. Linear regression parameters obtained by comparing VitD, $C K$ and reactive oxygen species (ROS). Deviation from horizontal was considered significant if the $P$ value was $<0.05$.

\begin{tabular}{ccccc}
\hline & Equation & $\mathbf{R}^{\mathbf{2}}$ & P Value & Deviation from Horizontal \\
\hline VitD vs. ROS & $-1.027^{*} \mathrm{X}+322.5$ & 0.045 & $<\mathbf{0 . 0 5}$ & SIGNIFICANT \\
\hline VitD vs. CK & $-1.529^{*} \mathrm{X}+391.1$ & 0.005 & 0.345 & NOT SIGNIFICANT \\
\hline VitD vs. T/C & $-0.009^{*} \mathrm{X}+1.543$ & 0.035 & $<\mathbf{0 . 0 5}$ & SIGNIFICANT \\
\hline CK vs. ROS & $0.537^{*} \mathrm{X}+187.6$ & 0.013 & 0.107 & NOT SIGNIFICANT \\
\hline CK vs. T/C & $-0.000^{*} \mathrm{X}+1.317$ & 0.017 & 0.071 & NOT SIGNIFICANT \\
\hline T/C vs. ROS & $0.000^{*} \mathrm{X}+1.213$ & 0.000 & 0.874 & NOT SIGNIFICANT \\
\hline
\end{tabular}




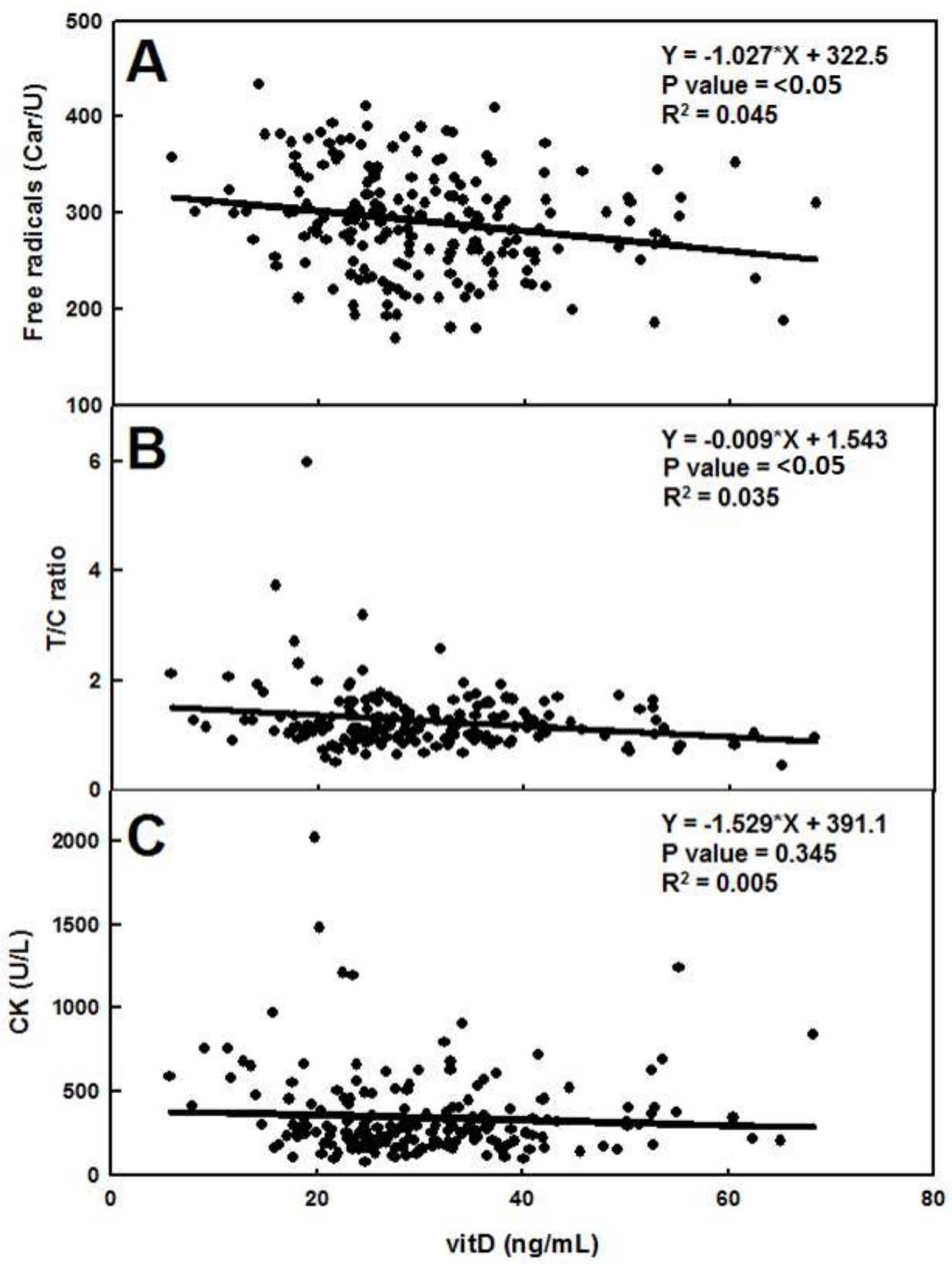

Figure 2. Linear correlation between vitD and free radicals (A), vitD and T/C (B), vitD and CK (C). For each regression the corresponding equation, the $\mathrm{R}^{2}$ and the $P$ value are shown.

\section{Discussion}

We retrospectively studied the psychophysical markers in elite soccer players during a competitive season in order to verify/recognize whether the large loads of physical activity carried out by professional athletes could raise concerns about their physical health. The $\mathrm{T} / \mathrm{C}$ ratio has been used as a marker of overtraining [31] based on the assumption that free testosterone is a marker of anabolism while cortisol is indicative of catabolism. Our results showed that although the averaged $\mathrm{T} / \mathrm{C}$ ratio values (above the 0.76 threshold limit during the whole season) were consistent with an appropriate training program, most of the athletes experienced high levels of CK and ROS. Moreover, despite the significant 
outdoor activity, during the "vitamin D winter", vitamin D values were often below the sufficiency level, compared with the general population.

The CK and ROS values were above the normal clinical range limit in $74.9 \%$ and $42.6 \%$ of the cases, respectively. These percentages became even higher ( $76.4 \%$ and $46.2 \%$ for CK and ROS, respectively) if samples taken from athletes on an injury period were omitted.

The CK serum activity, is a physical stress marker widely used in sport [32] but is also a marker of pathological conditions like acute myocardial infarction, myositis and myocarditis, hypothyroidism, myopathies etc. [33]. None of the athletes were affected by any of these conditions, however, CK activity might be above the normal clinical range in healthy subjects as well [34] due to CK leaking into the bloodstream upon muscular injury and, after strenuous physical activity. CK serum activity transiently rises to as much as 30 times the upper limit within 24 to $48 \mathrm{~h}$ and then slowly decreases over the next 7 days [35]. Thus, relatively high CK activity levels in professional athletes performing sports involving physical contacts, like soccer, could be considered as normal and are not necessarily associated to an overtraining condition. The highest percentages of samples above the $195 \mathrm{U} / \mathrm{L}$ limit, observed in the pre-seasons (between July and August) and at the beginning of the season (September to November), might be consistent with the impact to the newly started training program, after the rest period, and the consequent adaptation. If CK can be considered as a "pure" physical stress marker and does not induce any "side effect" to the athletes, the same is not true for ROS. Reactive oxygen species, also known as free radicals, are formed during the mitochondrial respiration, whose rate is enhanced during exercise, as mitochondrial superoxide or consequently to reperfusion after exercise-induced transient skeletal muscle ischemia [36]. ROS participate in a variety of chemical reactions and are also essential in adaptation to exercise (mitochondrial biogenesis, myofibers regeneration), however, when produced in excess, they can oxidize, and thus damage, a range of biological molecules, including lipids (e.g., membrane phospholipids), nucleic acids (DNA), as well as carbohydrates and proteins [37,38]. High ROS concentrations are associated with a decline in cognitive functions, as observed in some neurodegenerative disorders and age-dependent decay of neuroplasticity [38]. Thus, a long term exposure to this highly reactive species might lead to pathological implications [39]. Interestingly, one of the most common neurodegenerative disease among former professional soccer players is ALS [8] whose etiology is associated with the deposition of SOD1, a metalloenzyme responsible for scavenging free radicals [40], into insoluble aggregates in motor neurons. This amyloid-like formation is probably due to a structural destabilization and/or oxidative damage induced by gene mutations [15]. Because a high level of ROS results in higher transcription and translation of the SOD1 gene [41], we might speculate that an increased ROS level, constantly perpetuated along the athlete's career, will induce an almost constant SOD1 overexpression and thus promote the concentration-dependent formation of SOD1-amyloid-like aggregates [42] possibly associated with the pathogenesis of this neurodegenerative disease. In our study almost $50 \%$ of the measured levels were above the normal clinical limit, posing concerns for the long-term health of the players.

VitD, mainly synthesized by the skin when exposed to ultraviolet B radiation (UVB) [27], refers to a group of related steroid hormones involved in several physiological processes centered on the maintenance of calcium and phosphate homeostasis, as well as iron and zinc [27]. Since UVB is necessary to synthesize the vitD precursor cholecalciferol, vitD deficiency in populations living at high latitudes is common, especially during winter [30]. Although an optimal $(25(\mathrm{OH}) \mathrm{D})$ level helps to maintain the musculoskeletal system efficiency $[43,44]$, studies on athletes highlighted a surprisingly high prevalence of vitD insufficiency, both in outdoor and indoor disciplines $[45,46]$.

The average $(25(\mathrm{OH}) \mathrm{D})$ of the 29 athletes was, in the cold season, similar to those of the general population living at the same latitude. The four players of African origins do not lower significantly the averaged vitD levels, also, from recent population statistics [47] we expect a similar percentage of people of African origin in the general population living in the Milan area as well. Thus, the comparison between the whole group and the general population can be considered as pertinent. As previously observed in other studies [48] we noticed a scenario of insufficiency whilst deficiency was observed 
mainly in the four athletes of African origins, primarily due to skin pigmentation [49]. This was quite surprising because professional soccer players spend most of their time outdoors and, at this latitude even in the cold seasons, $2 \mathrm{~h}$ of sun exposure with $10 \%$ of body exposure at solar noon are sufficient for an optimal vitamin D dose [50]. This might be tentatively explained by the training outfit of the athletes which, in the cold season, usually covers a large part of the body. In contrast, during the warm season, where the training is usually performed with short sleeves and short pants, the athletes group showed averaged vitD levels much higher than the general population living at the same latitude.

Interestingly we found a significant correlation between $(25(\mathrm{OH}) \mathrm{D})$ and ROS (Table 1) which might confirm the recently discovered antioxidant role attributed to this hormone [51]. A weak yet significant correlation was found also between $(25(\mathrm{OH}) \mathrm{D})$ and the T/C ratio. Higher levels of vitD were thus associated to a slightly increased risk of overtraining. The correlation might indicate a possible biological interaction between the hormones, however, because of the rather high $P$ value as well as the lack of correlation when outliers were removed, further studies, involving larger dataset, are needed to investigate this issue.

Although no correlation between vitD status and athletic performance have been shown previously [45], and lower rates of osteoporotic fractures have been recorded in African-Americans having insufficient vitD levels [49], there are now evidences that vitD protects against other chronic conditions, including cardiovascular disease, diabetes, and some cancers [49]. The possible antioxidant effect observed in this study might be a further reason to suggest a vitD supplementation to professional athletes.

\section{Conclusions}

Although modern training programs seem to avoid the risk of overtraining in professional soccer players, others psychophysical stress markers, like the free radicals, are often above the normal clinical limit posing as a long term risk for several pathological situations like neurodegenerative diseases. Our data seems to associate an antioxidant effect with normal/high vitD levels. Thus, in the light of the general vitD insufficiency observed in the 29 professional soccer players, we suggest that vitD supplementation should become a general practice for professional soccer players and athletes that have to cope with high ROS levels for a long period of their life. Supplementation will preserve athletes from the harmful skeletal effects of low level of vitD as well as mitigate the detrimental high reactivity of ROS capable of damaging nucleic acids and protein conformation.

Supplementary Materials: The following are available online at http://www.mdpi.com/1660-4601/17/10/3484/s1, Table S1: VitD levels (ng/mL) measured during the soccer season 2017/2018. Table S2: CK levels (U/L) measured during the soccer season 2017/2018. Table S3: ROS levels (Car/U) measured during the soccer season 2017/2018. Table S4: T/C ratios measured during the soccer season 2017/2018.

Author Contributions: D.F. wrote the paper and contributed to data analysis. G.L. contributed to data analysis and writing of the paper. M.S., M.P. and, A.M. contributed to data collection. M.L. contributed to data analysis and paper supervising. All authors have read and agreed to the published version of the manuscript.

Funding: This research did not receive any specific grant from funding agencies in the public, commercial, or not-for-profit sectors.

Conflicts of Interest: The authors declare no conflict of interest.

\section{References}

1. Eigenschenk, B.; Thomann, A.; McClure, M.; Davies, L.; Gregory, M.; Dettweiler, U.; Inglés, E. Benefits of outdoor sports for society. A systematic literature review and reflections on evidence. Int. J. Environ. Res. Public Health 2019, 16, 937.

2. Lavie, C.J.; O'Keefe, J.H.; Sallis, R.E. Exercise and the heart-the harm of too little and too much. Curr. Sports Med. Rep. 2015, 14, 104-109. 
3. Meeusen, R.; Duclos, M.; Foster, C.; Fry, A.; Gleeson, M.; Nieman, D.; Raglin, J.; Rietjens, G.; Steinacker, J.; Urhausen, A. Prevention, diagnosis, and treatment of the overtraining syndrome: Joint consensus statement of the european college of sport science and the American College of Sports Medicine. Med. Sci. Sports Exerc. 2013, 45, 186-205.

4. Halson, S.L.; Jeukendrup, A.E. Does overtraining exist? An analysis of overreaching and overtraining research. Sports Med. 2004, 34, 967-981.

5. Adlercreutz, H.; Harkonen, M.; Kuoppasalmi, K.; Näveri, H.; Huhtaniemi, I.; Tikkanen, H.; Remes, K.; Dessypris, A.; Karvonen, J. Effect of training on plasma anabolic and catabolic steroid hormones and their response during physical exercise. Int. J. Sports Med. 1986, 7, 27-28.

6. Newsholme, E.A.; Parry-Billings, M.; McAndrew, N.; Budgett, R. A biochemical mechanism to explain some characteristics of overtraining. In Advances in Nutrition and Top Sport; Karger Publishers: Basel, Switzerland, 2015; pp. 79-93.

7. Patel, D.R.; Yamasaki, A.; Brown, K. Epidemiology of sports-related musculoskeletal injuries in young athletes in United States. Transl. Pediatr. 2017, 6, 160-166.

8. Mackay, D.F.; Russell, E.R.; Stewart, K.; MacLean, J.A.; Pell, J.P.; Stewart, W. Neurodegenerative disease mortality among former professional soccer players. N. Engl. J. Med. 2019, 381, 1801-1808. [PubMed]

9. Pearce, N.; Gallo, V.; McElvenny, D. Head trauma in sport and neurodegenerative disease: An issue whose time has come? Neurobiol. Aging 2015, 36, 1383-1389. [PubMed]

10. Blecher, R.; Elliott, M.A.; Yilmaz, E.; Dettori, J.R.; Oskouian, R.J.; Patel, A.; Clarke, A.; Hutton, M.; McGuire, R.; Dunn, R.; et al. Contact sports as a risk factor for amyotrophic lateral sclerosis: A systematic review. Glob. Spine J. 2019, 9, 104-118.

11. Beghi, E.; Logroscino, G.; Chiò, A.; Hardiman, O.; Millul, A.; Mitchell, D.; Swingler, R.; Traynor, B.J. Amyotrophic lateral sclerosis, physical exercise, trauma and sports: Results of a population-based pilot case-control study. Amyotroph. Lateral Scler. 2012, 11, $289-292$.

12. Belli, S.; Vanacore, N. Proportionate mortality of Italian soccer players: Is amyotrophic lateral sclerosis an occupational disease? Eur. J. Epidemiol. 2005, 20, 237-242. [PubMed]

13. Kuwahara, S.; Sato, Y. Retired American football players have a four times higher chance of getting Alzheimer disease or amyotrophic lateral sclerosis. Brain Nerve 2013, 65, 996-997. [PubMed]

14. Pansarasa, O.; Bordoni, M.; Diamanti, L.; Sproviero, D.; Gagliardi, S.; Cereda, C. Sod1 in amyotrophic lateral sclerosis: "Ambivalent" behavior connected to the disease. Int. J. Mol. Sci. 2018, 19, 1345.

15. McAlary, L.; Aquilina, J.A.; Yerbury, J.J. Susceptibility of mutant SOD1 to form a destabilized monomer predicts cellular aggregation and toxicity but not in vitro aggregation propensity. Front. Neurosci. 2016, 10, 499. [PubMed]

16. Silva, J.R.; Rumpf, M.C.; Hertzog, M.; Castagna, C.; Farooq, A.; Girard, O.; Hader, K. Acute and residual soccer match-related fatigue: A systematic review and meta-analysis. Sports Med. 2018, 48, 539-583. [PubMed]

17. Ascensão, A.; Rebelo, A.; Oliveira, E.; Marques, F.; Pereira, L.; Magalhães, J. Biochemical impact of a soccer match-Analysis of oxidative stress and muscle damage markers throughout recovery. Clin. Biochem. 2008, $41,841-851$.

18. Fatouros, I.G.; Chatzinikolaou, A.; Douroudos, I.I.; Nikolaidis, M.G.; Kyparos, A.; Margonis, K.; Michailidis, Y.; Vantarakis, A.; Taxildaris, K.; Katrabasas, I.; et al. Time-course of changes in oxidative stress and antioxidant status responses following a soccer game. J. Strength Cond. Res. 2010, 24, 3278-3286.

19. Romagnoli, M.; Sanchis-Gomar, F.; Alis, R.; Risso-Ballester, J.; Bosio, A.; Graziani, R.L.; Rampinini, E. Changes in muscle damage, inflammation, and fatigue-related parameters in young elite soccer players after a match. J. Sports Med. Phys. Fit. 2016, 56, 1198-1205.

20. Trecroci, A.; Porcelli, S.; Perri, E.; Pedrali, M.; Rasica, L.; Alberti, G.; Longo, S.; Iaia, F.M. Effects of different training interventions on the recovery of physical and neuromuscular performance after a soccer match. $J$. Strength Cond. Res. 2019. [CrossRef]

21. Dupont, G.; Nedelec, M.; McCall, A.; McCormack, D.; Berthoin, S.; Wisløff, U. Effect of 2 soccer matches in a week on physical performance and injury rate. Am. J. Sports Med. 2010, 38, 1752-1758.

22. Ekstrand, J.; Waldén, M.; Hägglund, M. A congested football calendar and the wellbeing of players: Correlation between match exposure of European footballers before the World Cup 2002 and their injuries and performers during that World Cup. Br. J. Sports Med. 2004, 38, 493-497. [PubMed] 
23. Banfi, G.; Marinelli, M.; Roi, G.S.; Agape, V. Usefulness of free testosterone/cortisol ratio during a season of elite speed skating athletes. Int. J. Sports Med. 1993, 14, 373-379.

24. Lombardi, G.; Vitale, J.A.; Logoluso, S.; Logoluso, G.; Cocco, N.; Cocco, G.; Cocco, A.; Banfi, G. Circannual rhythm of plasmatic vitamin D levels and the association with markers of psychophysical stress in a cohort of Italian professional soccer players. Chronobiol. Int. 2017, 34, 471-479. [PubMed]

25. Vitale, J.A.; Lombardi, G.; Cavaleri, L.; Graziani, R.; Schoenhuber, H.; Torre, A.L.; Banfi, G. Rates of insufficiency and deficiency of vitamin $\mathrm{D}$ levels in elite professional male and female skiers: A chronobiologic approach. Chronobiol. Int. 2018, 35, 441-449. [PubMed]

26. Kawamura, T.; Muraoka, I. Exercise-induced oxidative stress and the effects of antioxidant intake from a physiological viewpoint. Antioxidants 2018, 7, 119.

27. Ferrari, D.; Lombardi, G.; Banfi, G. Concerning the vitamin D reference range: Pre-analytical and analytical variability of vitamin D measurement. Biochem. Med. 2017, 27, 453-466.

28. Ferrari, D.; Ripa, M.; Premaschi, S.; Banfi, G.; Castagna, A.; Locatelli, M. LC-MS/MS method for simultaneous determination of linezolid, meropenem, piperacillin and teicoplanin in human plasma samples. J. Pharm. Biomed. Anal. 2019, 169, 11-18.

29. Ferrari, D.; Strollo, M.; Vidali, M.; Motta, A.; Pontillo, M.; Locatelli, M. Biochemical, immunochemical and serology analytes validation of the lithium heparin BD Barricor blood collection tube on a highly automated Roche COBAS8000 instrument. Acta Biomed. 2020, 91, 47-55.

30. Ferrari, D.; Lombardi, G.; Strollo, M.; Pontillo, M.; Motta, A.; Locatelli, M. Association between solar ultraviolet doses and vitamin D clinical routine data in European mid-latitude population between 2006 and 2018. Photochem. Photobiol. Sci. 2019, 18, 2696-2706.

31. Banfi, G.; Dolci, A. Free testosterone/cortisol ratio in soccer: Usefulness of a categorization of values. J. Sports Med. Phys. Fit. 2006, 46, 611-616.

32. Urhausen, A.; Gabriel, H.; Kindermann, W. Blood hormones as markers of training stress and overtraining. Sports Med. 1995, 20, 251-276. [PubMed]

33. Aujla, R.; Patel, R. Creatine Phosphokinase; StatPearls Publishing: Treasure Island, FL, USA, 2019.

34. Johnsen, S.H.; Lilleng, H.; Wilsgaard, T.; Bekkelund, S.I. Creatine kinase activity and blood pressure in a normal population: The Tromsø study. J. Hypertens. 2011, 29, 36-42. [PubMed]

35. Brewster, L.M.; Mairuhu, G.; Sturk, A.; van Montfrans, G.A. Distribution of creatine kinase in the general population: Implications for statin therapy. Am. Heart J. 2007, 154, 655-661. [PubMed]

36. Cooper, C.E.; Vollaard, N.B.; Choueiri, T.; Wilson, M.T. Exercise, free radicals and oxidative stress. Biochem. Soc. Trans. 2002, 30, 280-285.

37. Venditti, P.; Di Meo, S. The role of reactive oxygen species in the life cycle of the mitochondrion. Int. J. Mol. Sci. 2020, 21, 2173.

38. Beckhauser, T.F.; Francis-Oliveira, J.; De Pasquale, R. Reactive oxygen species: Physiological and physiopathological effects on synaptic plasticity. J. Exp. Neurosci. 2016, 2016, 23-48.

39. Song, P.; Zou, M.H. Roles of reactive oxygen species in physiology and pathology. In Atherosclerosis: Risks, Mechanisms, and Therapies; Wiley-Blackwell: Hoboken, NJ, USA, 2015; ISBN 9781118828533.

40. Ighodaro, O.M.; Akinloye, O.A. First line defence antioxidants-superoxide dismutase (SOD), catalase (CAT) and glutathione peroxidase (GPX): Their fundamental role in the entire antioxidant defence grid. Alex. J. Med. 2018, 54, 287-293.

41. Rojo, A.I.; Salinas, M.; Martín, D.; Perona, R.; Cuadrado, A. Regulation of Cu/Zn-superoxide dismutase expression via the phosphatidylinositol 3 kinase/Akt pathway and nuclear factor-kB. J. Neurosci. 2004, 24, 7324-7334.

42. Di Gregorio, S.E.; Duennwald, M.L. Yeast as a model to study protein misfolding in aged cells. FEMS Yeast Res. 2018, 18, foy054.

43. Abrams, G.D.; Feldman, D.; Safran, M.R. Effects of Vitamin D on skeletal muscle and athletic performance. J. Am. Acad. Orthop. Surg. 2018, 26, 278-285.

44. Zhang, L.; Quan, M.; Cao, Z.B. Effect of Vitamin D supplementation on upper and lower limb muscle strength and muscle power in athletes: A meta-analysis. PLOS ONE 2019, 14, e0215826.

45. Seo, M.W.; Song, J.K.; Jung, H.C.; Kim, S.W.; Kim, J.H.; Lee, J.M. The associations of vitamin D status with athletic performance and blood-borne markers in adolescent athletes: A cross-sectional study. Int. J. Environ. Res. Public Health 2019, 16, 3422. 
46. Lanteri, P.; Lombardi, G.; Colombini, A.; Banfi, G. Vitamin D in exercise: Physiologic and analytical concerns. Clin. Chim. Acta 2013, 415, 45-53. [PubMed]

47. Regione Lombardia Osservatorio Regionale per L'Integrazione e la Multietnicità. Available online: https: //www.ismu.org/osservatorio-regionale-orim-pubblicazioni/ (accessed on 10 March 2020).

48. Eskici, G. The importance of vitamins for soccer players. Int. J. Vitam. Nutr. Res. 2015, 85, 225-244.

49. Tylavsky, F.A.; Cheng, S.; Lyytikä, A.; Viljakainen, H.; Lamberg-Allardt, C. Symposium: Optimizing Vitamin $\mathrm{D}$ intake for populations with special needs: Barriers to effective food fortification and supplementation. $J$. Nutr. 2006, 136, 1130-1134.

50. Serrano, M.A.; Cañada, J.; Moreno, J.C.; Gurrea, G. Solar ultraviolet doses and vitamin D in a northern mid-latitude. Sci. Total Environ. 2017, 574, 744-750.

51. Mokhtari, Z.; Hekmatdoost, Z.; Nourian, M. Antioxidant efficacy of vitamin D. J. Parathyr. Dis. 2017, 5, 11-16.

(C) 2020 by the authors. Licensee MDPI, Basel, Switzerland. This article is an open access article distributed under the terms and conditions of the Creative Commons Attribution (CC BY) license (http://creativecommons.org/licenses/by/4.0/). 